\title{
Trade-Off Inflação-Desemprego e Bem-Estar Subjetivo com Amostragem Complexa
}

\section{Inflation-Unemployment Trade-off and Subjective Well-Being with Complex Sampling}

\author{
Andressa Suelen Eugênio ${ }^{a}$ \\ Elder Tiago da Costa Souza ${ }^{a}$ \\ Marcel de Toledo Vieira ${ }^{\mathrm{b}}$
}

\begin{abstract}
Resumo: Este trabalho analisa o nível de bem-estar dos brasileiros através de informações contidas na pesquisa de opinião pública Latinobarómetro. Com uma amostra de 11.767 brasileiros, estimam-se modelos logit ordenado considerando-se o plano amostral e uma possível heterogeneidade regional. Os resultados vão ao encontro da literatura nacional e internacional, em que as taxas de desemprego e inflação são relevantes na determinação do bem-estar, sendo que a primeira é relativamente mais importante para os brasileiros. Já a consideração do plano amostral mostrou-se relevante ao evitar valores superestimados e efeitos sobre os trade-off inflação-desemprego e uma inexistente diferença relativa à região Nordeste do Brasil.
\end{abstract}

Palavras-chave: Bem-estar subjetivo. Felicidade. Logit ordenado. Amostragem complexa.

\begin{abstract}
This paper analyzes the level of well-being of Brazilians through information contained in the Latinobarómetro public opinion survey. We use a sample of 11,767 Brazilians to estimate ordered Logit models, considering the sampling plan and a possible regional heterogeneity. The results are in line with the national and international literature, where the unemployment rate and inflation rate are relevant in determining well-being, and the unemployment rate is relatively more important for Brazilians. Already the consideration of the sample plan was relevant, avoiding overestimated values and effects on inflation-unemployment trade-off, and a nonexistent difference relative to the Brazilian region Nordeste.
\end{abstract}

Keywords: Subjective well-being. Happiness. Ordered logit. Complex sampling.

JEL Classification: D6; E3; E6.

\footnotetext{
a Universidade Federal de Juiz de Fora (UFJF), Departamento de Economia, Programa de Pós Graduação em Economia (PPGE). Juiz de Fora, Minas Gerais, Brasil.

b Universidade Federal de Juiz de Fora (UFJF), Departamento de Estatística. Juiz de Fora, Minas Gerais, Brasil.
} 


\section{1 lntrodução}

Mudanças na dinâmica das taxas de inflação e desemprego geram impactos (negativos e/ou positivos) sobre os agentes econômicos, o que leva a questionamentos empíricos sobre como o bem-estar pode ser influenciado por tais comportamentos (TELLA; MACCULLOCH; OSWALD, 2003). Na macroeconomia moderna, pode-se encontrar uma função de bem-estar, $W(p ; U)$, cujos argumentos são a taxa de inflação $(p)$ e a taxa de desemprego $(U)$. Vale mencionar que uma regra de política ótima não pode ser definida caso os parâmetros dessa função não sejam conhecidos (TELLA; MAC-CULLOCH; OSWALD, 2001).

A teoria microeconômica nos revela que a mensuração do nível de bem-estar individual pode ser inferida por meio das escolhas dos indivíduos, ou seja, de suas preferências reveladas. Em contraste com a economia neoclássica, outra fonte de medida de bem-estar seria coletar de forma direta os níveis de felicidade por meio de pesquisas de opinião. Com abordagens diferentes, pode-se questionar a superioridade de suas medidas de utilidade (CORBI; MENEZES-FILHO, 2006; TELLA; MACCULLOCH, 2007). Para muitos objetivos, o bem-estar reportado, também chamado de felicidade, é uma aproximação empírica satisfatória para a utilidade individual (DIENER, 2000). Ele permite captar o sentimento de felicidade considerando a apreciação da vida como um todo (olhando para o passado e para o futuro) e não apenas determinado momento da vida (por exemplo, o presente ou um fato específico) (VEENHOVEN, 1991).

Tais considerações micro e macroeconômicas direcionam para uma discussão que envolve a taxa de inflação e a taxa de desemprego associadas às pesquisas de bem-estar autorrelatadas pelos agentes econômicos. Essa discussão pode contribuir para um processo desinflacionário ótimo das economias. As funções microeconométricas de bem-estar podem variar de acordo com o objetivo central da pesquisa em questão (TELLA; MACCULLOCH; OSWALD, 2003). Porém, variáveis de controle tais como idade, status de relacionamento, status individual de trabalho, sexo, renda etc., estão entre as informações mais usadas na literatura (FERRER-I-CARBONELL; FRIJTERS, 2004; STEVENSON; WOLFERS, 2008; CLARK; OSWALD, 1994).

Como suporte, Woodford (2002) argumenta que uma vantagem importante do uso de um modelo baseado na otimização do setor privado para analisar as consequências de regras de política é que existe um critério de bem-estar natural no contexto de tal modelo, fornecido pelas preferências dos agentes privados que são exibidos no modelo estrutural. Tal abordagem baseada em utilidade para a análise de bem-estar tem sido padrão na teoria das finanças públicas e com crescente aceitação em análises de política monetária. Além disso, modelos baseados em otimização individual podem ser construídos de forma que, graças à presença de rigidezes nominais, permitam efeitos realistas da política monetária sobre variáveis reais. 
A derivação de um critério de bem-estar baseado na utilidade dessa maneira pode não apenas nos permitir justificar uma preocupação geral com a estabilidade de preços, mas também fornecer respostas exatas à questão levantada anteriormente sobre a formulação precisa da função de perda apropriada. Essas respostas dependem das suposições que são feitas sobre a estrutura da economia; por exemplo, elas dependem crucialmente da natureza das rigidezes nominais que estão presentes (WOODFORD, 2002).

No que tange à inferência estatística, a abordagem clássica fundamenta-se na amostra aleatória simples, método que requer que cada membro da população tenha uma chance igual e independente de ser selecionado. Contudo, cabe mencionar que as pesquisas de opinião pública, tais como Latinobarómetro, Eurobarometer e World Values Survey, que abordam sobre o bem-estar individual, não selecionam suas amostras a partir de amostras aleatórias simples. Isso se dá, em parte, por restrições orçamentárias, por serem pesquisas internacionais (o que acarreta uma grande extensão territorial) e por limite de tempo. Em decorrência disso, outros métodos probabilísticos são geralmente utilizados em pesquisas de base populacional, como a amostragem estratificada e a amostragem por conglomerados em múltiplos estágios com probabilidades desiguais de seleção, para prover uma amostra representativa da população em tempo hábil e com orçamento plausível.

A combinação de vários métodos probabilísticos de amostragem para seleção de uma amostra representativa da população é chamada de desenho complexo de amostragem. Os efeitos dos desenhos amostrais complexos, se não considerados na estimação, podem levar a desvios padrões, intervalos de confiança e níveis de significância que não representam bem a amostra em questão. Isso ocorre porque as fórmulas comuns de análise estatística nos pacotes de estimação usuais são baseadas, em geral, nas hipóteses de observações que seguem um processo independente e identicamente distribuído (IID), ou de forma análoga, de amostragem aleatória simples com reposição (AASC) (PESSOA; SILVA, 1998). Além disso, a variabilidade dos pesos produz impactos tanto na estimação pontual quanto na estimação das variâncias dessas estimativas, que sofre ainda influência da estratificação e conglomeração.

Destarte, o impacto das simplificações feitas ao utilizar procedimentos e pacotes usuais de análise de dados gera resultados inapropriados. Com o intuito de mitigar esses resultados inapropriados, fazem-se necessários ajustes nos desenhos amostrais de modo a incorporar na análise os aspectos mencionados. Ao fazê-lo, a comparação dos resultados das análises realizadas por meio das duas formas permite avaliar o impacto de ignorar o plano amostral na análise dos dados resultantes de pesquisas amostrais complexas.

Nesse sentido, o presente trabalho é guiado pelas seguintes motivações, a saber: a) utilização da pesquisa de opinião pública Latinobarómetro, referente aos anos de 2003 a 2013; b) estimativa do trade-off inflação-desemprego brasileiro 
usando-se o modelo logit ordenado, assumindo-se que sua variância seja Var [eijxi] = p2 = 31; c) maior robustez dos resultados levando-se em consideração o plano amostral da pesquisa; e d) diante das enormes desigualdades regionais presentes no Brasil, investigação de diferenciais regionais de percepção de níveis de bem-estar em relação à taxa de inflação e taxa de desemprego.

De forma geral, o propósito é caracterizar o bem-estar individual dos brasileiros afetado pelas dinâmicas das relevantes variáveis macroeconômicas (taxa de inflação e taxa de desemprego), sempre controlando-se o modelo por meio de um vetor de características individuais. As contribuições para a literatura encontram-se na utilização de uma significativa amostra (cerca de 11.800 indivíduos) cujos dados são pouco explorados na economia brasileira, nos cuidados com o plano amostral usando a técnica de amostragem complexa na estimação do modelo e nas investigações complementares de cunho regional.

Além da presente introdução, o trabalho se organiza da seguinte forma: a segunda seção apresenta uma breve discussão do embasamento do modelo empírico, bem como os modelos propostos para estimação; a terceira expõe as metodologias, a base Latinobarómetro e os dados utilizados; a quarta discute os resultados alcançados; e, por fim, a quinta tece as considerações finais, salientando as contribuições e os principais resultados deste trabalho.

\section{Revisão de Literatura}

De acordo com a hipótese de expectativas racionais, fatores econômicos, como a taxa de inflação, não deveriam afetar em longo prazo as decisões dos indivíduos e, portanto, seu nível de satisfação. Para isso, algumas hipóteses são necessárias, tais como: ausência de assimetria no acesso à informação entre as pessoas e os agentes econômicos; todos os envolvidos têm total e completo acesso às informações sobre desemprego e inflação. Entretanto, uma vez afetadas as decisões, busca-se observar o quanto de taxa de desemprego seria necessário para compensar o aumento de um ponto percentual da taxa de inflação, ou vice-versa. (FREY; STUTZER, 2002).

A abordagem seguida por Rotemberg e Woodford (1997) se concentra no fato de que, quando os ajustes de preços são escalonados, a inflação induz a uma volatilidade espúria nos preços de algumas empresas em relação a outras, reduzindo a capacidade do sistema de preços de alocar recursos eficientemente. De tal forma, os autores desenvolvem um modelo no qual as relações estruturais são baseadas na otimização do comportamento individual e as empresas devem ocasionalmente manter seus preços fixos, resultando em distorções substanciais de preços relativos quando a inflação aumenta. Como discutido por Woodford (1999), suas estimativas para os Estados Unidos implicam um valor para os custos da inflação em relação ao desemprego da ordem de 20 , dado que o hiato do 
produto é medido em pontos percentuais e a inflação é medida a uma taxa anual anualizada. Ou seja, o peso sobre a inflação é 20 vezes o peso sobre o hiato do produto no bem-estar da sociedade.

Ainda, Rotemberg e Woodford (1997) explicam que, a partir de sua abordagem, exigir que as relações estruturais de uma pessoa sejam derivadas da otimização individual também tem a vantagem de se poder usar evidências de outras fontes sobre a natureza dos problemas que os indivíduos enfrentam para corroborar as especificações quantitativas usadas para explicar as relações entre séries temporais agregadas. Em última análise, seria a única maneira pela qual a "equivalência observacional" de uma infinidade de possíveis interpretações estruturais alternativas dos comovimentos de séries agregadas pode ser resolvida (ROTEMBERG; WOODFORD, 1997).

Os autores Clark e Oswald (1994), Stutzer e Lalive (2004), L. Winkelmann e R. Winkelmann (1998) sugerem que, em média, um aumento de um ponto percentual na taxa de desemprego é mais prejudicial para a satisfação com a vida do que um aumento de um ponto percentual na taxa de inflação. Sendo assim, diante da existência de um trade-off entre essas duas variáveis, é necessário uma queda de mais de um ponto percentual na taxa de inflação para compensar um aumento de um ponto percentual na taxa de desemprego.

Ao utilizar uma amostra de aproximadamente 250 mil indivíduos americanos e europeus, Tella, MacCulloch e Oswald (2001) observam que a taxa de desemprego, relativamente à taxa de inflação, é quase duas vezes mais prejudicial para a felicidade. Tal resultado é reforçado por Wolfers (2003) ao mostrar que, usando uma amostra de 504.581 pessoas em 16 países no período de 1973 a 1998, um aumento de 10 pontos percentuais na taxa de desemprego reduziria a felicidade média em 0.35 desvio padrão, enquanto que um aumento de 10 pontos percentuais na taxa de inflação reduziria a felicidade média em 0.07 desvio padrão. Por sua vez, Tella e MacCulloch (2006), com um modelo probit ordenado transformando pontuações de felicidade em números contínuos para dados de países da Organização para a Cooperação e Desenvolvimento Econômico (OCDE) ao longo do período 19751992, apresentam resultados de que um aumento de um ponto percentual na taxa de desemprego seria 4,7 vezes mais prejudicial para a felicidade do que um ponto percentual adicional na taxa de inflação.

Assim como Wolfers (2003), Blanchflower e Freeman (2007) estimam os coeficientes da função de bem-estar social para cerca de 500 mil indivíduos americanos e o leste europeu. Os resultados também vão na direção de que a taxa de desemprego teria um peso maior que a taxa de inflação sobre a felicidade dos indivíduos para tal amostra, sendo a taxa marginal de substituição estimada em -1,62. Outro resultado empírico interessante é o fato de que pessoas com nível educacional menor, bem como os idosos, estariam mais preocupados com o desemprego do que com o aumento do nível geral de preços. Contudo, os mais jovens e os de 
maiores níveis de escolaridade atribuem maior peso à taxa de inflação. Isso sugere que a consideração da estrutura demográfica da sociedade importa quando se deseja captar as percepções dos indivíduos no que diz respeito aos efeitos da taxa de inflação e da taxa de desemprego sobre seu bem-estar.

Com dados de pesquisas de bem-estar subjetivo para a América Latina, Ruprah e Luengas (2011) mostram que a taxa de desemprego seria cerca de oito vezes mais importante que a taxa de inflação, o que sugere uma maior sensibilidade relativamente aos países desenvolvidos. Todavia, observam importantes diferenças entre grupos testados, tais como o fato de que os mais jovens e cidadãos que dizem ter preferências políticas à esquerda dão maior preferência para o emprego do que à alta de preços.

Estudos mais recentes, como, por exemplo, o de Blanchflower et al. (2013), continuam a destacar os impactos negativos das altas nos preços e na taxa de desemprego sobre a felicidade dos indivíduos, bem como a maior sensibilidade em relação ao desemprego. Para tanto, os autores utilizaram uma amostra de mais de um milhão de europeus entre os anos de 1975 a 2012. Eles modificaram o índice de miséria desenvolvido por Arthur Okun ao introduzirem a razão de miséria pelo fato do primeiro subestimar a perda de bem-estar. Os novos resultados mostram que um aumento na taxa de desemprego de um ponto percentual diminui o bem-estar por duas vezes e meia a mais do que um aumento de um ponto percentual na taxa de inflação. Blanchflower e Posen (2014) descrevem que o desemprego, na verdade, reduz cinco vezes mais a felicidade que a taxa de inflação, sugerindo uma tolerância social maior relativamente à inflação.

Com o intuito de captar especificamente a sensibilidade dos indivíduos brasileiros com relação à dinâmica das variáveis macroeconômicas agregadas, taxa de inflação e taxa de desemprego, o presente trabalho propõe exercícios empíricos por meio do modelo logit ordenado. A escolha desse modelo se justifica porque considera a característica qualitativa e ordinal da variável satisfação com a vida obtida. Implicitamente, assume-se que as medidas de utilidade direta são uma proxy razoável do bem-estar dos brasileiros. Assim, o modelo empírico a ser estimado pode ser expresso pelo modelo 1:

$$
\text { Satvita }_{t i}=\beta_{1} \text { Desemprego }_{t}+\beta_{2} \text { inflacao }_{t}+\beta_{3 k} X_{t k i}+\beta_{4} \text { tempo }+\varepsilon_{t i},
$$

tal que Satvita ${ }_{t i}$ é a proxy utilizada para o bem-estar do indivíduo $i$ no ano $t$. Essa variável é tratada de forma equivalente à felicidade e à satisfação com a vida. $\mathrm{O}$ Desemprego ${ }_{t}$ é o percentual de pessoas desempregadas no ano $t$ e inflacao ${ }_{t}$ refere-se à taxa de inflação medida pela variação percentual do IPCA no ano t. $\mathrm{X}_{t k i}$ é um vetor de variáveis de controle de $k$ características pessoais retiradas do Latinobarómetro, tais como situação de emprego, estado civil, sexo, idade e idade ao quadrado, quartis de renda, nível educacional, tamanho da cidade onde o indivíduo reside, entre outras. O tempo é uma variável que busca captar alterações em cada ano, uma vez que não é 
possível acompanhar os indivíduos ao longo do tempo. O componente $\varepsilon_{t i}$ é o termo de erro aleatório nıe segue uma distribuição simétrica logística com média zero e variância igual a $\frac{\pi^{2}}{3}$.

De forma semelhante ao modelo 1, propõe-se uma análise adicional com o intuito de captar heterogeneidades regionais quanto aos efeitos das taxas de desemprego e inflação nacionais sobre o nível de satisfação do indivíduo da região j ( $j$ = Norte, Nordeste, Sul, Sudeste e Centro-Oeste), região à qual ele pertencia no momento da pesquisa. Para tanto, criaram-se variáveis dummies de intercepto. Outro exercício executado foi o de substituir as taxas de inflação e desemprego nacional, usadas na equação 1, pelas respectivas taxas de cada região. Essas propostas de análise são apresentadas por meio das equações 2 e 3, doravante denominadas de modelo II e modelo III, respectivamente.

$$
\begin{aligned}
& \text { Satvita }_{t i j}=\beta_{5} \text { Desemprego }_{t}+\beta_{6} \text { inflacao }_{t}+\beta_{7 j} Z_{t i j}+\beta_{8} W_{t i j}+\beta_{9 k} X_{t k i j}+\beta_{10} \text { tempo }+\varepsilon_{t i}, \\
& \text { Satvita }_{t i j}=\beta_{11} \text { Desemprego }_{t j}+\beta_{12} \text { inflacao }_{t j}+\beta_{13} X_{t i j}+\beta_{14} \text { tempo }+\varepsilon_{t i j}
\end{aligned}
$$

tal que Satvita $a_{t j}$ é a proxy utilizada para o bem-estar do indivíduo $i$, no ano $t$, que vive na região $j . Z_{t i j}$ é um vetor com as variáveis dummies de intercepto entre as taxas de desemprego e as $j$ regiões do país. $\mathrm{W}_{t i j}$ é um vetor com as variáveis dummies de intercepto entre a taxa de inflação e as $j$ regiões brasileiras; $X_{t k j}$ é um vetor de controle que inclui $k$ características pessoais retiradas do Latinobarómetro (incluindo situação de emprego, estado civil, sexo, idade e idade ao quadrado, quartis de renda, nível educacional, tamanho da cidade, etc.). No caso da equação 3, o Desemprego ${ }_{t j}$ é a taxa de desemprego no ano $t$ da região $j$, e a é a taxa de inflação no ano $t$ da região $j$.

Os modelos propostos são baseados nos trabalhos de Tella, MacCulloch e Oswald (2001), Wolfers (2003), Tella e MacCulloch (2006) e Corbi e Menezes-Filho (2006). Embora esses trabalhos tenham estudado de forma diversa e específica a problemática da satisfação como uma medida de bem-estar e quais fatores a impactam, eles contribuíram de forma direta e indireta para a seleção das variáveis explicativas utilizadas no presente trabalho. De acordo com a literatura, espera-se que os coeficientes relativos às variáveis taxa de desemprego e taxa de inflação sejam negativos e estatisticamente significativos. Após essa discussão do referencial empírico, a próxima seção introduz o método de estimação (logit ordenado), o plano amostral e os dados utilizados. 


\section{Modelo Logit Ordenado}

O modelo logit ordenado é utilizado para a análise de resultados e respostas ordenadas, categóricas e escolhas não quantitativas (GREENE; HENSHER, 2010). A justificativa para seu uso no presente trabalho é a variável dependente "satisfação com a vida”, tratada como uma variável categórica e qualitativa, dividida em quatro níveis: nada satisfeito $=1$; pouco satisfeito $=2$; satisfeito $=3$; e muito satisfeito $=4$. O modelo de escolha ordenada parte de um modelo de utilidade aleatória subjacente ou modelo de regressão latente, definido pela equação 4:

$$
\mathrm{y}^{*}{ }_{i}=\beta^{\prime} \chi_{i}+\varepsilon_{i} ; i=1 ; \ldots ; n ;
$$

na qual $x$ não inclui um intercepto e a utilidade latente ou "medida", , é observada na forma discreta, ou seja:

$$
\begin{aligned}
y_{i}^{*} & =1 \text { se } y_{i}^{*} \leq \mu_{1} \\
& =2 \text { se } \mu_{i} \leq y_{1}^{*} \leq \mu_{2} \\
& =\vdots \\
& =\text { J se } \mu_{J-1} \leq y_{1}^{*} \leq \mu_{j}
\end{aligned}
$$

O vetor $X_{i}$ é um conjunto de $K$ variáveis que são assumidas como independentes de $\varepsilon_{i} ; \beta$ é um vetor de $K$ parâmetros, que é o objeto de estimação e inferência; as $n$ observações da amostra são rotuladas $i=1 ; \ldots ; n$, e assume-se que tanto os coeficientes quanto os parâmetros de limite não diferem entre os indivíduos.

O modelo contém as utilidades marginais desconhecidas, $\beta$, assim como $j-1$ parâmetros de limite desconhecidos, $\mu_{j}$, todos a serem estimados utilizando uma amostra de $n$ observações, indexados por $i=1, \ldots, j$. Os dados consistem das variáveis $x_{i}$ e do resultado discreto observado, $\mathrm{y}_{i}=0 ; 1, \ldots, j$. Os pressupostos convencionais sobre as propriedades do termo de erro, $\varepsilon_{i}$, completam as especificações do modelo, a saber: $\varepsilon_{i}$ é um erro aleatório com uma função de distribuição cumulativa $(f d c), F\left(\varepsilon_{i} \mid X\right)=F\left(\varepsilon_{i}\right)$, e com densidade $\mathrm{f}\left(\varepsilon_{i}\right)=F^{\prime}\left(\varepsilon_{i}\right)$. A suposição da distribuição de $\varepsilon_{i}$ inclui a independência ou a exogeneidade de, $X_{i}$.

Diferentemente dos modelos de regressão comumente usados, que descrevem a relação direta entre variável dependente e variáveis explicativas, o modelo logit ordenado descreve as probabilidades de resposta entre $y_{i}$ e as variáveis $X_{i}$. Assim, pelas leis de probabilidade, as probabilidades associadas com os resultados observados são:

$$
\operatorname{Prob}\left[y_{i}=j \mid x_{i}\right]=\operatorname{Prob}\left[\varepsilon_{i} \leq \mu_{j}-\beta^{\prime} x_{i}\right]-\operatorname{Prob}\left[\mu_{j-1}-\beta^{\prime} x_{i}\right], j=0,1, \ldots, J .
$$


Segundo Greene e Hensher (2010), algumas normalizações se fazem necessárias para a identificação dos parâmetros do modelo. Em primeiro lugar, a fim de preservar os sinais positivos de todas as probabilidades, exige-se que $\mu_{j}>\mu_{j-l}$. Em segundo lugar, se o suporte utilizado é a reta real, então $\mu_{0}=-\infty$ e $\mu_{j}=+\infty$. Uma vez que os dados não contêm informações incondicionais sobre a escala da variável subjacente (se $y_{i}$ é escalonado por qualquer valor positivo, então, escalonando o desconhecido $\mu_{j}$ e $\beta$ pelo mesmo valor, preserva-se os resultados observados), um parâmetro de variância incondicional, $\operatorname{Var}\left[\varepsilon_{i}\right]=\sigma_{\varepsilon}^{2}$, não é identificado (estimável). Usa-se, convenientemente, a restrição de se identificar $\sigma_{\varepsilon}$ igual a uma constante, $\bar{\sigma}$. A aproximação usual para essa normalização em modelos logit ordenado é assumir que $\operatorname{Var}\left[\varepsilon_{i} \mid x\right]=\frac{\pi^{2}}{3}$.

Jackman (2000) apresenta outras duas possibilidades de restrição de identificação, apresentadas no Quadro 1. Ressalta-se que neste trabalho as restrições impostas estão apresentadas na linha 2.

Quadro 1 - Identificação de possíveis restrições

\begin{tabular}{cccc}
\hline & $\beta$ & $\sigma$ & $\mu$ \\
\hline 1 & Irrestrito. & Fixa-se, por exemplo, $\sigma=1$ & Fixa-se um $\mu_{j^{\prime}}$, por exemplo, \\
& & ou $\sigma=\pi^{2} / 3$. & \\
& & Fixa-se, por exemplo, $\sigma=1$ & Irrestrito. \\
2 & Exclui-se o intercepto. & ou . & Fixa-se dois $\mu_{j s^{\prime}}$ \\
& & Irrestrito. & \\
\hline
\end{tabular}

Fonte: Elaboração própria a partir de Jackman (2000) e Greene e Hensher (2010).

Com o conjunto de normalizações completo, a função de verossimilhança para estimar os parâmetros do modelo é baseada nas probabilidades implícitas:

$$
\operatorname{Prob}\left[y_{i}=j \mid x_{i}=\left[F\left(\mu_{j}-\beta^{\prime} x_{i}\right)-F\left(\mu_{j-1}-\beta^{\prime} x_{i}\right)\right]>0 ; j=0,1, \ldots, J\right.
$$

A estimação dos parâmetros é um problema direto de estimação de máxima verossimilhança (EMV), tal que o objetivo desse estimador, por meio de um modelo ordenado, é encontrar as estimativas de e que maximizem a probabilidade conjunta de obter os valores observados (CAMERON; TRIVEDI, 2005). O logaritmo da função de verossimilhança pode ser apresentado como:

$$
\log L=\sum_{i=1}^{n} \sum_{j=0}^{j} m_{i j} \log \left[F\left(\mu_{j}-\beta^{\prime} x_{i}\right)-F\left(\mu_{j-1}-\beta^{\prime} x_{i}\right)\right]>0 \quad j=0,1, \ldots, J .
$$

em que é uma função indicadora que obtém o resultado um caso a função dentro do colchete for verdadeira e zero caso contrário. A maximização é sujeita às restrições e . Como não há uma função média condicional a analisar, normalmente se 
usa as próprias probabilidades para interpretar os parâmetros, isto é, os efeitos marginais. Os efeitos marginais no modelo de escolha ordenados podem ser calculados por:

$$
\delta_{j}\left(x_{i}\right)=\frac{\partial \operatorname{Prob}\left(y=j \mid x_{i}\right)}{\partial x_{i}}=\left[f\left(\mu_{j-1}-\beta^{\prime} x_{i}\right)-f\left(\mu_{j}-\beta^{\prime} x_{i}\right)\right] \beta
$$

Porém, de acordo com Greene e Hensher (2010), para uma variável dummy o efeito parcial é obtido usando uma diferença de probabilidades, ao invés de uma derivada. Deixando D ser uma variável dummy do modelo a ser estimado e , o seu coeficiente, pode-se obter o efeito da mudança em $D$ de zero para um com todas as outras variáveis mantidas em seus valores de interesse (por exemplo, suas médias):

$$
\Delta_{j}(\mathrm{D})=\left[\mathrm{F}\left(\mu_{j}-\beta^{\prime} x_{i}+\gamma\right)-\mathrm{F}\left(\mu_{j-1}-\beta^{\prime} x_{i}+\gamma\right)\right]-\left[\mathrm{F}\left(\mu_{j}-\beta^{\prime} x_{i}\right)-F\left(\mu_{j-1}-\beta^{\prime} x_{i}\right)\right]
$$

A implicação do resultado anterior é que o efeito da mudança em uma das variáveis no modelo depende dos parâmetros, dos dados e de qual probabilidade é de interesse.

\subsection{Amostragem em Pesquisas Transversais}

Uma característica relevante da análise de dados amostrais é o desenho amostral utilizado pelo estatístico amostrista para a seleção da amostra da pesquisa. O desenho amostral de uma pesquisa pode ser definido como um procedimento adotado para selecionar uma amostra, ou um subconjunto, de uma população alvo, ou população finita (VIEIRA, 2012).

O desenho amostral utilizado pelo Latinobarómetro para seleção da amostra de 11.767 indivíduos brasileiros ao longo dos anos de 2003 a 2013, usada no presente trabalho, é baseado em uma amostra probabilística em três etapas. Há, também, a inclusão de uma quarta etapa não probabilística, que considera cotas de entrevistados. Somado a isso, o desenho amostral é composto por um erro amostral de $+/-2,8 \%$ para um intervalo de confiança de $95 \%$ com $100 \%$ de representatividade da população brasileira com 16 anos ou mais. Ainda, a amostragem é feita de acordo com as proporcionalidades do censo, ou seja, $85 \%$ da população urbana e $15 \%$ da população rural.

A amostragem utilizada pela pesquisa nas três primeiras etapas é do tipo estratificada, ou seja, há a divisão da população em estratos (mutuamente exclusivos e exaustivos) de acordo com características conhecidas. A partir desses extratos, são selecionadas unidades (normalmente de forma independente), chamadas de unidades primárias e/ou secundárias de amostragem. A amostra representativa da população no Latinobarómetro foi estratificada com alocação proporcional ao tamanho da população de cada estrato, tal que os estratos são os 26 estados brasileiros e o Distrito Federal. 
Dentro de cada estrato, as amostras probabilísticas de aglomerados são selecionadas conforme segue:

a) primeira etapa: seleção dos municípios: método de probabilidade proporcional ao tamanho (PPT);

b) segunda etapa: setores censitários de seleção: método PPT;

c) terceira etapa: seleção de casas; e

d) quarta etapa: seleção de entrevistados: ação proporcional por sexo, idade, educação e ocupação (cotas).

Ressalta-se que, devido à disponibilidade na base de dados, apenas a primeira etapa de seleção é considerada na estimação dos modelos. No entanto, isso não é visto como um problema, dado que a primeira etapa é a mais representativa na estimação utilizando amostragem complexa (PESSOA; SILVA, 1998).

\subsection{Dados}

Para melhor entendimento da base de dados utilizada para desenvolver este trabalho, esta seção será dividida em duas subseções: descrição da base de dados Latinobarómetro e variáveis do modelo.

\subsection{Apresentação da Base de Dados Latinobarómetro}

O Latinobarómetro é uma pesquisa de opinião pública anual que envolve cerca de 20.000 entrevistas por ano em 18 países da América Latina, o que representa mais de 600 milhões de habitantes. A corporação Latinobarómetro pesquisa o desenvolvimento da democracia e das economias, bem como as sociedades, por meio de indicadores de opinião, atitudes, comportamentos e valores.

Em 1995, o Latinobarómetro realizou o primeiro trabalho de campo para pesquisas na América Latina e incluiu oito países: Argentina, Brasil, Chile, México, Paraguai, Peru, Uruguai e Venezuela. A partir de 1996, o estudo foi realizado em 17 países e, já em 2004, a República Dominicana foi inserida na pesquisa, completando os 18 países latino-americanos (com exceção de Cuba). Até o momento, ocorreram 19 pesquisas na América Latina, o que resultou em 354.268 entrevistas. A pesquisa mais recente foi realizada em 2016 e aplicada a 20.204 entrevistados entre 15 de maio e 15 de junho de 2016, com amostras representativas de $100 \%$ da população em cada um dos 18 países, o que abrange 597 milhões de habitantes.

O Brasil foi contemplado em todos os anos em que a pesquisa foi realizada e, ao longo desses anos, totalizou 19.316 entrevistas em todas as grandes regiões do país. Contudo, nos anos 1995, 1996, 1998, 1999 e 2002, a pergunta sobre o nível de satisfação com a vida não estava incluída no questionário da pesquisa. Apenas a partir de 2003 essa pergunta passou a ser incluída em todos os anos da pesquisa. Portanto, os dados utilizados neste trabalho serão a partir de tal ano. 
Além da base de dados supracitada, a próxima subseção faz a apresentação das variáveis do modelo e de mais duas fontes de dados que foram utilizadas na obtenção das variáveis macroeconômicas (inflação, inflação por região, desemprego e desemprego por região).

\subsection{Variáveis do Modelo}

O objetivo central deste estudo é observar como o bem-estar autorrelatado dos brasileiros é afetado pela taxa de inflação e taxa de desemprego. Para tanto, utilizou-se a pesquisa Latinobarómetro de forma a extrair informações sobre a satisfação com a vida (variável dependente) e características sociodemográficas. A Figura 1 ilustra a distribuição da satisfação com a vida dos brasileiros no que se refere ao período entre 2003 e 2013. Observa-se que cerca de 70\% dos brasileiros tendem a relatar que estão satisfeitos com a vida que levam. Somado a isso, tem-se apenas 3\% dos indivíduos que dizem estar completamente insatisfeitos, 7\% que relatam estar plenamente satisfeitos e o restante $20 \%$ pouco satisfeitos.

Figura 1 - Distribuição da satisfação com a vida (Brasil)

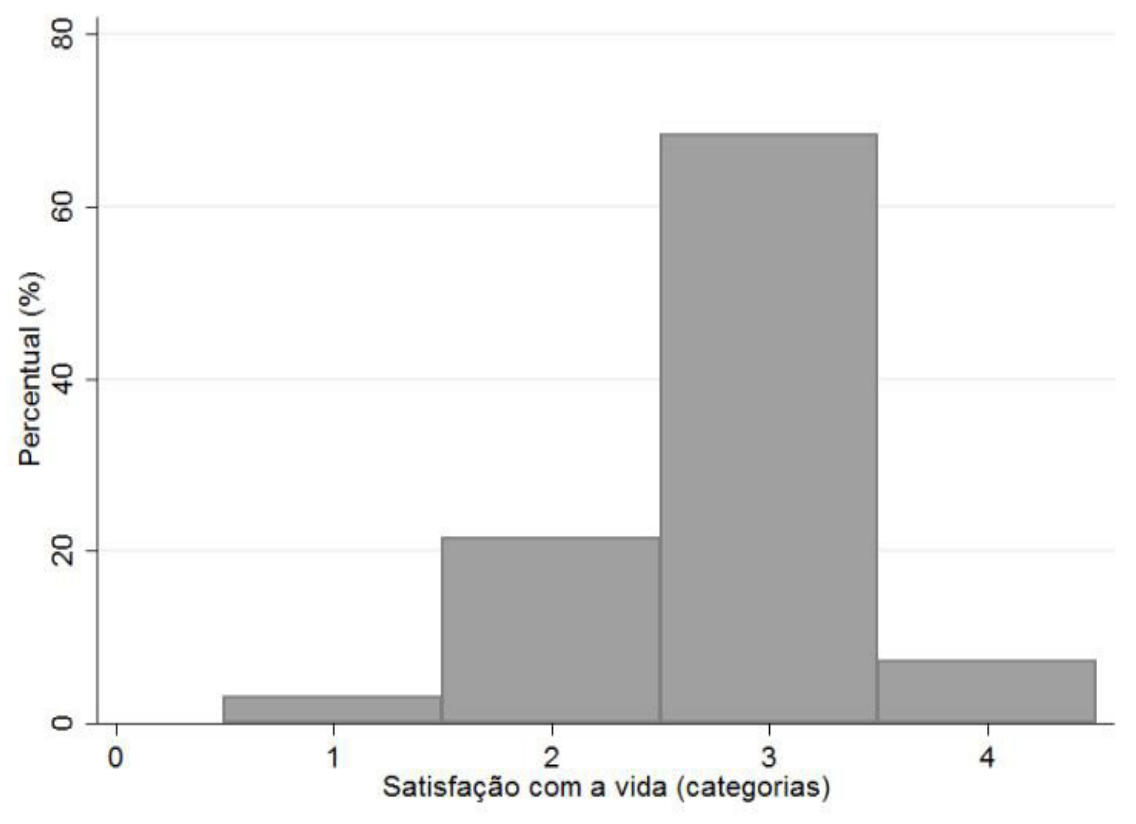

Fonte: Elaboração própria a partir de dados do Latinobarómetro (2018).

A taxa de inflação medida pela variação do Índice Nacional de Preços ao Consumidor Amplo (IPCA) agregado e por região é obtida no Sistema Gerencia- 
dor de Séries Temporais (BCB, 2018) do Banco Central do Brasil, cuja fonte é o Instituto Brasileiro de Geografia e Estatística, Sistema Nacional de Índices de Preços ao Consumidor (IBGE/SNIPC).

Por fim, a Pesquisa Nacional por Amostra de Domicílios (PNAD), realizada pelo IBGE (2018) é utilizada para obter as taxas de desemprego agregado, bem como as taxas de desemprego por região do Brasil. De acordo com o IBGE (2018), a taxa de desocupação, medida em porcentagem ao ano, corresponde à percentagem das pessoas desocupadas em relação às pessoas economicamente ativas.

\section{Resultados Empíricos}

A Tabela 1 apresenta os resultados estimados para os parâmetros da equação 1 pela EMV com a amostra de 11.767 observações supracitadas. Nessa regressão foram registradas quatro interações para que o máximo da função de log-verossimilhança fosse atingido (-9927.2767) e, pelo teste de Wald (distribuição qui-quadrada com 27 parâmetros), rejeitou-se os efeitos nulos de interações dos parâmetros (Prob $\left.>X^{2}=0,0000\right)$ a um nível de significância de 1\%. Somado a isso, encontra-se um pseudo $R^{2}$ de MacFaldden de 0,0492. As estimativas dos pontos de corte revelam que:

$$
\begin{aligned}
& y=1 \text { se } y^{*} \leq-5.629503 \\
& y=2 \text { se }-5.629503 \leq y^{*} \leq-3.176835 \\
& y=3 \text { se }-3.176835 \leq y^{*} 0.7520784 \\
& y=4 \text { se } y^{*}>0.7520785
\end{aligned}
$$

Tabela 1 - Regressão por EMV: estimativa da probabilidade do bem-estar dos brasileiros

\begin{tabular}{lcccccc}
\hline & \multicolumn{3}{c}{ Sem plano amostral } & \multicolumn{5}{c}{ Com plano amostral } \\
\hline Variáveis & Coeficientes & $\begin{array}{c}\text { Desvio } \\
\text { padrão }\end{array}$ & Prob & Coeficientes & $\begin{array}{c}\text { Desvio } \\
\text { padrão }\end{array}$ & Prob \\
\hline Segundo quartil & 0.5148075 & 0.065993 & 0.000 & 0.509725 & 0.0730913 & 0.000 \\
Terceiro quartil & 1.171126 & 0.0683929 & 0.000 & 1.166487 & 0.0772507 & 0.000 \\
Quarto quartil & 1.557746 & 0.0843077 & 0.000 & 1.55489 & 0.0937994 & 0.000 \\
Homem & 0.1046744 & 0.0433315 & 0.016 & 0.1072 & 0.0398524 & 0.007 \\
Idade & -0.0363173 & 0.0072727 & 0.000 & -0.03621 & 0.0065733 & 0.000 \\
Idade $^{2}$ & 0.0004542 & 0.0000816 & 0.000 & 0.000452 & 0.0000738 & 0.000 \\
Casado $^{\text {Divorciado }}$ & 0.1142023 & 0.0520431 & 0.028 & 0.114995 & 0.0524191 & 0.029 \\
& -0.2647305 & 0.0798756 & 0.001 & -0.26886 & 0.0873827 & 0.002 \\
\hline
\end{tabular}


conclusão.

\begin{tabular}{|c|c|c|c|c|c|c|}
\hline \multirow[b]{2}{*}{ Variáveis } & \multicolumn{2}{|c|}{ Sem plano amostral } & \multicolumn{4}{|c|}{ Com plano amostral } \\
\hline & Coeficientes & $\begin{array}{l}\text { Desvio } \\
\text { padrão }\end{array}$ & Prob & Coeficientes & $\begin{array}{l}\text { Desvio } \\
\text { padrão }\end{array}$ & Prob \\
\hline $\begin{array}{l}\text { Empregado } \\
\text { público }\end{array}$ & 0.1835363 & 0.0848272 & 0.030 & 0.183977 & 0.0906033 & 0.043 \\
\hline $\begin{array}{l}\text { Empregado } \\
\text { privado }\end{array}$ & 0.195538 & 0.0556337 & 0.000 & 0.195587 & 0.050243 & 0.000 \\
\hline Desempregado & -0.5443155 & 0.0817143 & 0.000 & -0.5457 & 0.0757785 & 0.000 \\
\hline Estudante & -0.0291745 & 0.0864754 & 0.736 & -0.02769 & 0.0795477 & 0.728 \\
\hline Aposentado & -0.0020988 & 0.0669736 & 0.975 & -0.00446 & 0.0595176 & 0.940 \\
\hline Dona de casa & 0.248701 & 0.1116992 & 0.026 & 0.248236 & 0.1141396 & 0.030 \\
\hline Primário & -0.0085088 & 0.0585778 & 0.885 & -0.00959 & 0.0620746 & 0.877 \\
\hline Médio & -0.1142425 & 0.0537951 & 0.034 & -0.11417 & 0.0601785 & 0.058 \\
\hline Superior & 0.2239169 & 0.103122 & 0.030 & 0.217188 & 0.1012244 & 0.032 \\
\hline $\begin{array}{l}\text { Cidades 5-10 mil } \\
\text { habitantes }\end{array}$ & -0.0474526 & 0.124387 & 0.703 & -0.05066 & 0.1955205 & 0.796 \\
\hline $\begin{array}{l}\text { Cidades 10-20 mil } \\
\text { habitantes }\end{array}$ & 0.0568543 & 0.1099917 & 0.605 & 0.052271 & 0.1624135 & 0.748 \\
\hline $\begin{array}{l}\text { Cidades 20-40 mil } \\
\text { habitantes }\end{array}$ & -0.0891079 & 0.107147 & 0.406 & -0.08992 & 0.153467 & 0.558 \\
\hline $\begin{array}{l}\text { Cidades } 40-50 \text { mil } \\
\text { habitantes }\end{array}$ & -0.2946196 & 0.1146841 & 0.010 & -0.29795 & 0.163545 & 0.069 \\
\hline $\begin{array}{l}\text { Cidades 50-100 mil } \\
\text { habitantes }\end{array}$ & -0.2830076 & 0.1010518 & 0.005 & -0.28444 & 0.1458456 & 0.052 \\
\hline $\begin{array}{l}\text { Cidades } 100 \text { mil } \\
\text { habitantes ou mais }\end{array}$ & -0.4508873 & 0.0986506 & 0.000 & -0.45323 & 0.1498685 & 0.003 \\
\hline Capital & -0.1568876 & 0.1124772 & 0.163 & -0.15841 & 0.1492135 & 0.289 \\
\hline Tempo & -0.0429232 & 0.0150816 & 0.004 & -0.04294 & 0.0200141 & 0.032 \\
\hline $\begin{array}{l}\text { Taxa de inflação } \\
\text { agregada }\end{array}$ & -2.799695 & 1.314755 & 0.033 & -2.88171 & 1.722778 & 0.095 \\
\hline $\begin{array}{l}\text { Taxa de } \\
\text { desemprego } \\
\text { agregado }\end{array}$ & -20.62756 & 4.366413 & 0.000 & -20.7598 & 5.930614 & 0.001 \\
\hline
\end{tabular}

Fonte: Elaboração própria a partir de dados do Latinobarómetro (2018).

Ainda na Tabela 1, observam-se coeficientes, bem como os desvios padrões, distintos para as diferentes estimações considerando-se ou não o plano amostral. Para as variáveis de controle, seus coeficientes são ora superestimados (como, por exemplo, as variáveis de quartil de renda), ora subestimadas (como, por exemplo, o sexo) quando o plano amostral não é considerado, como pode-se analisar comparando-se a segunda e quinta colunas da Tabela 1. Já as variáveis de taxa de inflação 
e taxa de desemprego têm seus respectivos coeficientes reduzidos quando o plano amostral é considerado na estimação, refletindo menores efeitos sobre o bem-estar dos brasileiros. No entanto, o sentido de impacto das variáveis sobre a satisfação com a vida dos indivíduos, bem como a significância estatística não se alteram com a presença do plano amostral na estimação.

Em termos econômicos, os coeficientes dos quartis de renda sugerem que um aumento na renda do indivíduo acarreta um aumento na probabilidade deste relatar um maior nível de satisfação com a vida, ou seja, o nível de renda é positivamente correlacionada com a satisfação com a vida. Porém, apesar da relação positiva, a probabilidade se reduz quando se considera o quartil superior. De igual forma, Frey e Stutzer (2002), Deaton (2008) e Stevenson e Wolfers (2013) obtiveram resultados comuns que argumentam que o impacto marginal de uma unidade monetária adicional vai reduzindo à medida que a renda aumenta, quando esta atinge certo nível até que mais renda tem efeito pequeno ou nulo sobre o bem-estar.

Outro resultado que merece destaque é quanto ao status de emprego. Controlando-se a situação de emprego por "autonômo", nota-se que estar desempregado traz significativa insatisfação para os brasileiros, inclusive mantendo as demais variáveis constantes como a renda. Segundo L. Winkelmann e R. Winkelmann (1998), além da perda de renda que acompanha uma situação de desemprego, há também custos não monetários, sociais e psicológicos que acarretam uma grande queda no nível de bem-estar. No que se refere à idade e à idade ao quadrado, apesar de pouco expressivos, os resultados sugerem que o aumento de um ano de vida diminui a probabilidade de se atingir um maior nível de satisfação, evidências corroboradas por Clark e Oswald (1994) e Corbi e Menezes-Filho (2006), que propõem um padrão convexo no formato de "U" para a satisfação com a vida. Nesse caso, as pessoas de meia idade (por volta de 40 anos) tendem a apresentar um menor nível de satisfação do que os jovens e os mais velhos, tudo mais constante. Outras variáveis de controle levadas em consideração, tais como ser autônomo, estar aposentado, possuir apenas o nível primário de escolaridade, residir em cidades de 5 a 40 mil habitantes e em capitais não apresentaram significância estatística de seus coeficientes. Conforme esperado, os resultados mostram que tanto a taxa de inflação quanto a taxa de desemprego afetam negativamente a probabilidade de um indivíduo relatar um maior nível de satisfação, logo, independentemente de considerar ou não o plano amostral, a taxa de desemprego é vista como mais prejudicial que os aumentos de preços.

Greene e Hensher (2010) chamam atenção para o fato de que os modelos de escolha ordenada geram probabilidades de resposta e, portanto, não descrevem diretamente a relação entre as variáveis $y_{i}$ e $x_{i}$. Desse modo, faz-se necessário o uso dos efeitos marginais para a interpretação dos impactos na variável dependente. Um efeito marginal (EM), ou efeito parcial, na maioria das vezes, mede o efeito condicional na 
média de , dada uma mudança em um dos regressores, $\beta_{j}$. Em outras palavras, ele capta a mudança na variável dependente $(y)$ por unidade de mudança na variável independente $(x)$. No modelo de regressão linear, o EM é igual ao coeficiente de inclinação, o que simplifica a análise. Para modelos não lineares, necessita-se um método mais complexo para o cálculo de EMs. Em vista que há um efeito marginal para cada indivíduo na amostra, pode-se apresentar três formas de se realizar o cálculo dos efeitos marginais, a saber: a) o efeito marginal médio (média dos efeitos marginais em cada $x=x_{i}$ ); b) o efeito marginal na média (efeito marginal em $\mathrm{x}=\overline{\mathrm{x}}$ ); e c) o efeito marginal em um valor representativo (efeito marginal em $x=x^{*}$ ). No presente trabalho, adota-se a segunda opção para cada categoria de resposta da variável dependente, ou seja, estimam-se os efeitos marginais na média de cada variável independente sobre as probabilidades preditas de cada resposta ordenada (CAMERON; TRIVEDI, 2009).

Seguindo o proposto, foram calculados os efeitos marginais na média para cada probabilidade predita de categoria de resposta em que os indivíduos: são nada satisfeitos (1); pouco satisfeitos (2); satisfeitos (3); e muito satisfeitos (4). No entanto, os sinais e coeficientes das categorias intermediárias podem nem sempre representar a direção do efeito, devido à logística padrão da função densidade de probabilidade ser simétrica em torno de zero e declinar monotonicamente à medida que o argumento cresce em valor absoluto (WOOLDRIDGE, 2010). Portanto, analisam-se, a partir da Tabela 2, os efeitos marginais para as categorias das extremidades, isto é, nada satisfeitos e muito satisfeitos. Essas categorias apresentam, respectivamente, a probabilidade de 2,48\% e 6,23\%, sem plano amostral, e 2,47\% e 6,22\%, com plano amostral, de indivíduos que relataram pertencer a cada uma delas.

Tabela 2 - Efeitos marginais calculado na média

\begin{tabular}{lcccc}
\hline \multirow{2}{*}{ Variáveis } & \multicolumn{4}{c}{ Categorias } \\
\cline { 2 - 5 } & \multicolumn{3}{c}{ Nada satisfeito (1) } & Muito satisfeito (4) \\
\hline & Sem & Com & Sem & Com \\
& plano amostral & plano amostral & plano amostral & plano amostral \\
\cline { 2 - 5 } Taxa de inflação & 0.0677879 & 0.0695519 & -0.1636239 & -0.1682394 \\
Taxa de desemprego & 0.499447 & 0.5010508 & -1.205546 & -1.211994 \\
\hline
\end{tabular}

Fonte: Elaboração própria.

Quanto aos sinais dos efeitos marginais das variáveis por categoria apresentados na Tabela 2, pode-se ver que, diante de um aumento nas taxas de inflação e/ou na taxa de desemprego, aumenta-se a probabilidade das pessoas relatarem uma menor satisfação com a vida, isso pode ser observado através do sinal positivo apresentado na categoria (1). De forma análoga, como há um deslocamento de indivíduos entre as categorias de satisfação, observa-se que a categoria (4) apresenta 
um sinal negativo no seu coeficiente. Isso revela que um aumento nas mesmas variáveis macroeconômicas impacta negativamente a probabilidade do indivíduo em declarar o mais alto nível de bem-estar.

Não menos importante é analisar a magnitude dos coeficientes. Primeiramente, observa-se que os brasileiros dão pesos diferentes para a taxa de inflação e a taxa de desemprego, dado a categoria de satisfação na qual se encontra. Comparando-se, por exemplo, a terceira e quinta colunas da Tabela 2, tem-se que os indivíduos que relataram estar "muito satisfeitos" dão um maior peso às essas variáveis macroeconômicas do que aqueles que disseram "nada satisfeitos". Considerando-se um aumento de um ponto percentual na taxa de desemprego média, ou seja, de 8,10\% para 9,10\%, tem-se uma diminuição da satisfação com a vida em 0,01205546 ponto percentual (p.p.), considerando-se o plano amostral e a quarta categoria de satisfação. Desconsiderado o plano amostral, ao repetir o exercício aumentando a taxa de desemprego em um p.p., observa-se uma redução de 0,0121994 ponto percentual na satisfação. Já o impacto do aumento de um ponto percentual na média da inflação, de 5,88\% para 6,88\%, é de 0,001636239 com o plano amostral e 0,001682394 sem considerar o plano.

Apesar de o efeito do aumento na taxa de desemprego sobre a satisfação parecer pequeno, não é desprezível. Ao considerar a amostra de 11.767 indivíduos, nota-se que o aumento desloca cerca de 140 brasileiros pertencentes à categoria de satisfação máxima com a vida para categorias inferiores, nas quais a probabilidade de um indivíduo reportar o maior nível de satisfação passa de 6,23\% para 5,02\%. Já o mesmo aumento na taxa de inflação diminuiria a probabilidade de 19 indivíduos reportarem o maior nível de satisfação com a vida. A Figura 2 apresenta a curva da taxa de desemprego mais inclinada que a curva da taxa de inflação, corroborando os resultados encontrados nos exercícios empíricos.

Figura 2 - Efeito marginal das taxas de desemprego e inflação sobre a satisfação com a vida

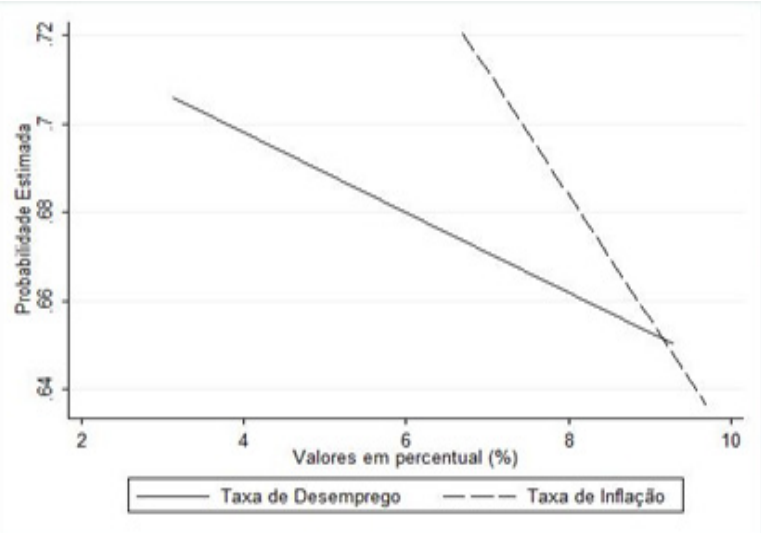

Fonte: Elaboração própria. 
De acordo com a teoria macroeconômica, existe consenso de que a curva de Phillips é vertical no longo prazo. No entanto, torna-se importante analisar não somente as preferências/pesos dos indivíduos mas também o trade-off desemprego-inflação de curto prazo, de forma a sugerir um melhor caminho desinflacionário. Seguindo-se a literatura, estima-se o trade-off entre desemprego e inflação, mantendo-se a utilidade constante. Conforme Corbi e Menezes-Filho (2006), assume-se que a utilidade seja linear no intervalo relevante, de forma que a margem torna-se igual à média. Com esse pressuposto, o que se está medindo é a inclinação da curva de indiferença, ou seja, a taxa marginal de substituição entre taxa de desemprego e taxa de inflação.

Desconsiderando-se o plano amostral, o trade-off inflação-desemprego é estimado em $\frac{0,499447}{0,0677879}=7,37$. Tal resultado revela que um aumento de um ponto percentual na taxa de desemprego poderia ser compensado (em termos de satisfação com a vida) com uma queda de 7,37 pontos percentuais na taxa de inflação. Ao se considerar o plano amostral, o trade-off é estimado em $\frac{0,5010508}{0,0695519}=7,20$.

De forma geral, os resultados mostraram um trade-off maior do que o encontrado por Corbi e Menezes-Filho (2006) para a economia brasileira $(3,38)$ e por Tella e MacCulloch (2006) para a OCDE (4,7). Entretanto, são próximos aos valores encontrados por Ruprah e Luengas (2011), em cujo estudo a taxa de desemprego é cerca de oito vezes mais importante que a taxa de inflação para uma amostra da América Latina. Contudo, todos os resultados apontam para a mesma direção que o desemprego, em termos de satisfação com a vida, é mais custoso aos indivíduos do que a inflação.

\subsection{Heterogeneidade Regional}

A Tabela 3 apresenta os efeitos marginais da estimação para os modelos regionais propostos. 


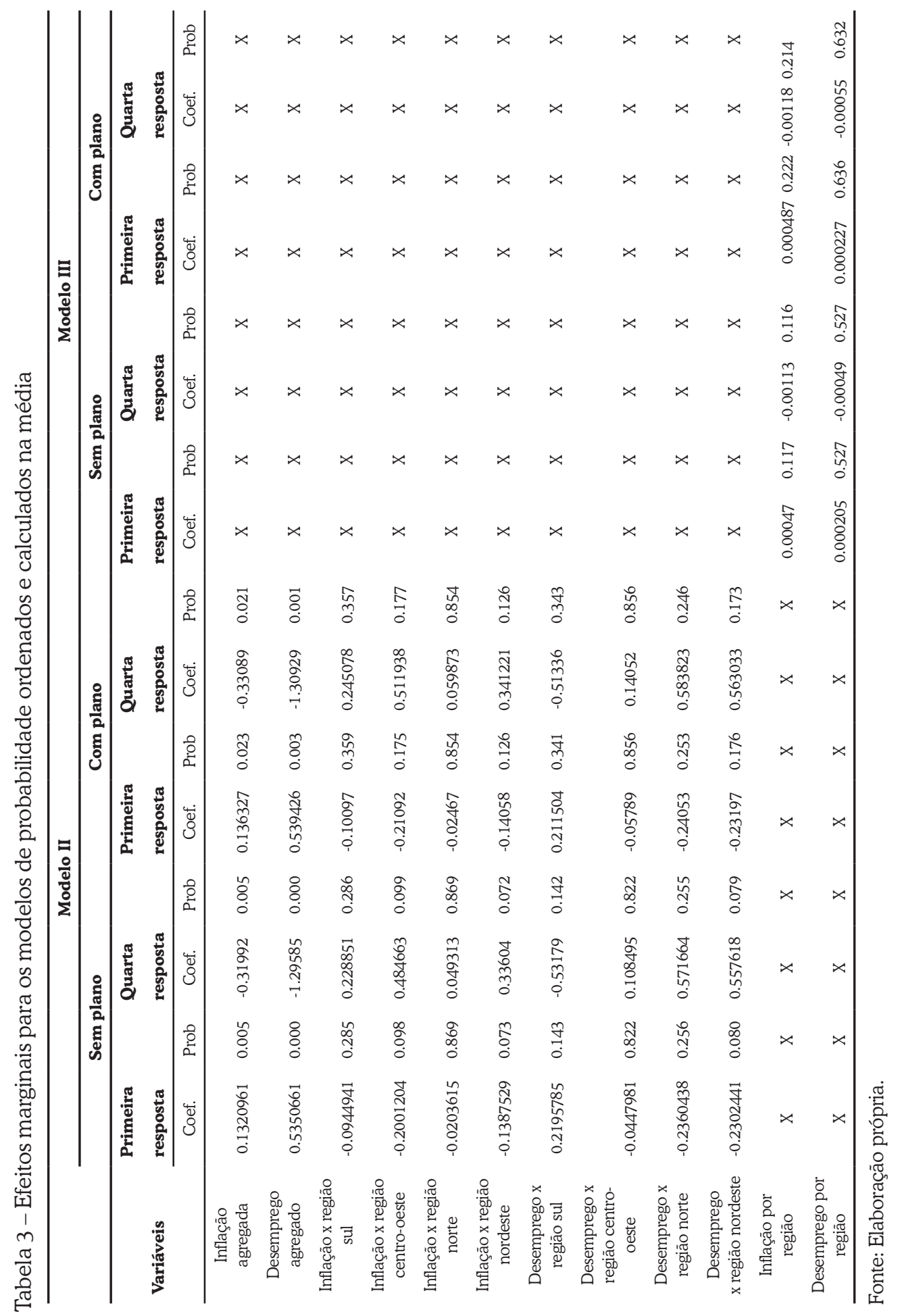

Análise Econômica, Porto Alegre, v. 38, n. 76, p. 145-170, jun. 2020. 
Considerando-se o modelo II, os parâmetros estimados para a taxa de inflação e a taxa de desemprego agregados continuam estatisticamente significativos e com os sinais esperados. Quando se analisam os resultados desconsiderando o plano amostral, nota-se que as variáveis dummies de intercepto relacionadas à região Nordeste apresentam-se positivas e estatisticamente significativas, ao nível de 10\% de significância. Tal resultado revela que os indivíduos que vivem nessa região enfrentariam um trade-off diferenciado dos demais indivíduos do país.

Para avaliar como os indivíduos da região Nordeste têm sua satisfação impactada de forma diferenciada pela taxa de desemprego, por exemplo, usa-se a soma dos coeficientes da taxa de desemprego e da variável de dummy de intercepto. Tal valor é estimado em 1,29585 + 0,079 = 1,21685. De forma análoga, tem-se o valor de 0,24792 para a soma entre a taxa de inflação e a respectiva variável dummy de intercepto. Esses resultados mostram que, para os indivíduos residentes na região Nordeste, seria necessário uma queda de, aproximadamente, cinco pontos percentuais (resultante da seguinte fração: $\frac{-1,21586}{-0,24792}$ ) na taxa de inflação para compensar, em termos de satisfação com a vida, um aumento de um ponto percentual na taxa de desemprego, enquanto que, para os residentes das demais regiões do país, esse valor seria de quatro pontos percentuais. Porém, quando se considera o plano amostral na estimativa do modelo II, os resultados mostram ausência de significância estatística para as variáveis dummies de intercepto, revelando efeitos de não existir heterogeneidade entre os brasileiros.

No caso do modelo III, no qual se considera as taxas regionais de desemprego e inflação, os resultados vão na direção de efeitos semelhantes, seja considerando-se ou não o plano amostral, e indicam que os indivíduos não observam as taxas de desemprego e inflação da sua respectiva região. Ou seja, o nível de satisfação não apresenta influência das taxas de desemprego e inflação regional, sugerindo que os brasileiros prestam maior atenção nas variáveis agregadas nacionais, talvez pela sua maior divulgação e discussão.

De forma geral, os resultados chamam atenção para a importância da utilização do plano amostral na estimação de modelos que levem em consideração dados oriundos de pesquisas de opinião, pois, caso o plano amostral não fosse considerado, além de valores superestimados, levariam à conclusão de que os nordestinos enfrentam um trade-off inflação-desemprego diferenciado dos demais brasileiros.

\section{Considerações Finais}

O presente trabalho visou analisar o nível de bem-estar dos brasileiros por meio de informações sobre sua satisfação com a vida fornecida na pesquisa de opinião pública Latinobarómetro. Essa pesquisa se destaca por conter dados de diversos países, inclusive da América Latina, há cerca de 20 anos. Partindo-se de uma 
amostra de 11.767 indivíduos brasileiros, estimam-se modelos logit ordenado com o intuito de se observar como as taxas de inflação e desemprego impactam o nível de bem-estar no Brasil. De forma a tornar os resultados mais robustos, considera-se o plano amostral na discussão, uma vez que sua ausência pode influenciar os resultados. ${ }^{1}$ De forma complementar, avalia-se uma possível heterogeneidade de bem- estar no que diz respeito às regiões do país. Os modelos são baseados em uma especificação microeconométrica de bem-estar padrão, conforme destacado.

Os resultados vão ao encontro da literatura empírica nacional e internacional no que diz respeito ao fato de as variáveis taxa de desemprego e taxa de inflação serem relevantes na determinação do bem-estar. ${ }^{2}$ Ainda, a taxa de desempregado torna-se mais importante para os brasileiros do que a taxa de inflação quando se diz respeito ao seu bem-estar. Outro resultado interessante é a influência positiva da renda, mas menor quando se consideram quartis superiores. A literatura destaca que o impacto marginal de uma unidade monetária adicional diminui à medida que a renda aumenta, como apresentado por Deaton (2008) e Stevenson e Wolfers (2013).

Já a consideração do plano amostral mostrou-se relevante, evitando valores super ou subestimados e efeitos consequentes sobre o trade-off inflação-desemprego, evitando, assim, uma inexistente diferença relativa à região Nordeste.

Resumidamente, o trabalho mostra um trade-off maior do que o encontrado por Corbi e Menezes-Filho (2006) para a economia brasileira $(3,38)$ e por Tella e MacCulloch (2006) para OCDE (4,7), mas próximo ao valor encontrado por Ruprah e Luengas (2011), em que a taxa de desemprego é cerca de oito vezes mais importante que a taxa de inflação para uma amostra da América Latina. Contudo, todos os resultados apontam para a mesma direção em que o desemprego, em termos de satisfação com a vida, é mais custoso aos indivíduos, inclusive para os brasileiros, do que é a taxa de inflação. Logo, existirá sempre um maior custo político quando o Banco Central do Brasil necessitar exercer uma política monetária mais restritiva para reduzir e estabilizar os preços na economia, uma vez que esse órgão considera a estabilidade de preços uma condição necessária, mas não suficiente, para o crescimento econômico sustentado.

\section{Referências}

BCB. Sistema gerenciador de séries temporais (SGS). 2018. Disponível em: https://www3. bcb.gov.br/sgspub/localizarseries/localizarSeries.do?method=prepararTelaLocalizarSeries. Acesso em: 03 jun. 2020.

BLANCHFLOWER, D. G. et al. The effects of macroeconomic shocks on well-being. Manuscript: University of Stirling, 2013.

$1 \quad$ Ver Vieira (2012).

2 Ver Tella, MacCulloch e Oswald (2001), Wolfers (2003), Tella e MacCulloch (2006) e Corbi e Menezes-Filho (2006). 
BLANCHFLOWER, D. G.; FREEMAN, R. B. Youth employment and joblessness in advanced countries. Chicago: University of Chicago Press, 2007.

BLANCHFLOWER, D. G.; POSEN, A. S. Wages and labor market slack: making the dual mandate operational. Peterson Institute for International Economics Working Paper, n. 14-6, 2014.

CAMERON, A. C.; TRIVEDI, P. K. Microeconometrics: methods and applications. Cambridge: Cambridge University Press, 2005.

CAMERON, A. C.; TRIVEDI, P. K. Microeconomics using stata. Lakeway Drive: Stata Press Books, 2009.

CLARK, A. E.; OSWALD, A. J. Unhappiness and unemployment. The Economic Journal, v. 104, n. 424, p. 648-659, 1994.

CORBI, R. B.; MENEZES-FILHO, N. A. Os determinantes empíricos da felicidade no Brasil. Brazilian Journal of Political Economy, v. 26, n. 4 (104), 2006.

DEATON, A. Income, health and wellbeing around the world: evidence from the gallup world poll. Journal of economic perspectives, v. 22, n. 2, p. 53, 2008.

DIENER, E. Subjective well-being: the science of happiness and a proposal for a national index. American Psychological Association, v. 55, n. 1, 2000.

FERRER-I-CARBONELL, A.; FRIJTERS, P. How important is methodology for the estimates of the determinants of happiness?* The Economic Journal, v. 114, n. 497, p. 641-659, 2004.

FREY, B. S.; STUTZER, A. The economics of happiness. World Economics, v. 3, n. 1, p.1-17, 2002.

GREENE, W. H.; HENSHER, D. A. Modeling ordered choices: a primer. Cambridge: Cambridge University Press, 2010.

IBGE. Pesquisa nacional por amostra de domicílios (PNAD). 2018. Disponível em: https://www. ibge.gov.br/. Acesso em: 03 jun. 2020.

JACKMAN, S. Models for ordered outcomes political science 200c. Political Science, v. 150, p. 350C, 2000.

LATINOBARÓMETRO. Latinobarómetro opinión pública latinoamericana. 2018. Disponível em: http://www.latinobarometro.org/lat.jsp. Acesso em: 03 jun. 2020.

PESSOA, D. G. C.; SILVA, P. L. N. Análise de dados amostrais complexos. São Paulo: Associação Brasileira de Estatística, 1998. v. 1.

ROTEMBERG, J. J.; WOODFORD, M. An optimization-based econometric framework for the evaluation of monetary policy. NBER Macroeconomics Annual, v. 12, p. 297-346, 1997.

RUPRAH, I. J.; LUENGAS, P. Monetary policy and happiness: preferences over inflation and unemployment in Latin America. The Journal of Socio-Economics, v. 40, n. 1, p. 59-66, 2011. 
STEVENSON, B.; WOLFERS, J. Economic growth and subjective well-being: reassessing the Easterlin paradox. NBER Working Paper, n. 14282, 2008.

STEVENSON, B.; WOLFERS, J. Subjective well-being and income: is there any evidence of satiation? NBER Working Paper, n. 18992, 2013.

STUTZER, A.; LALIVE, R. The role of social work norms in job searching and subjective wellbeing. Journal of the European Economic Association, v. 2, n. 4, p. 696-719, 2004.

TELLA, R. D.; MACCULLOCH, R. Some uses of happiness data in economics. The Journal of Economic Perspectives, p. 25-46, 2006.

TELLA, R. D.; MACCULLOCH, R. Happiness, contentment and other emotions for central banks. National bureau of economic research, 2007. Disponível em: https:/www.nber.org/papers/w13622. Acesso em: 03 jun 2020.

TELLA, R. D.; MACCULLOCH, R. J.; OSWALD, A. J. Preferences over inflation and unemployment: evidence from surveys of happiness. American Economic Review, v. 91, n. 1, p. 335-341, 2001.

TELLA, R. D.; MACCULLOCH, R. J.; OSWALD, A. J. The macroeconomics of happiness. Review of Economics and Statistics, v. 85, n. 4, p. 809-827, 2003.

VEENHOVEN, R. Is happiness relative? Social Indicators Research, v. 24, n. 1, p. 1-34, 1991.

VIEIRA, M. T. A consideração da amostragem complexa na análise de dados longitudinais. In: LIVRO de minicurso da 57 reunião da RBras, Piracicaba: ESALQ-USP, 2012.

WINKELMANN, L.; WINKELMANN, R. Why are the unemployed so unhappy? evidence from panel data. Economica - UC, v. 65, n. 257, p. 1-15, 1998.

WOLFERS, J. Did unilateral divorce laws raise divorce rates? A reconciliation and new $r$ esults. NBER Working Paper, n. 10014, 2003.

WOODFORD, M. Optimal monetary policy inertia. The Manchester School, v. 67, p. 1-35, 1999.

WOODFORD, M. Inflation stabilization and welfare. Contributions in Macroeconomics, v. 2, n. $1,2002$.

WOOLDRIDGE, J. M. Econometric analysis of cross section and panel data. Cambridge: The MIT Press, 2010. 


\section{Apêndice A}

Tabela 4 - Resultados para os modelos de região

\begin{tabular}{|c|c|c|c|c|c|c|c|c|}
\hline \multirow[b]{3}{*}{ Variáveis } & \multicolumn{4}{|c|}{ Modelo I } & \multicolumn{4}{|c|}{ Modelo II } \\
\hline & \multicolumn{2}{|l|}{ Sem plano } & \multicolumn{2}{|l|}{ Com plano } & \multicolumn{2}{|l|}{ Sem plano } & \multicolumn{2}{|l|}{ Com plano } \\
\hline & Coeficiente & Prob & Coeficiente & Prob & Coeficiente & Prob & Coeficiente & Prob \\
\hline rendasuff2 & 0.542146 & 0.000 & 0.536798 & 0.000 & 0.521784 & 0.000 & 0.516604 & 0.000 \\
\hline rendasuff3 & 1.21407 & 0.000 & 1.209199 & 0.000 & 1.180497 & 0.000 & 1.175651 & 0.000 \\
\hline rendasuff4 & 1.602481 & 0.000 & 1.599552 & 0.000 & 1.573223 & 0.000 & 1.57013 & 0.000 \\
\hline homem & 0.103473 & 0.017 & 0.106007 & 0.008 & 0.10353 & 0.017 & 0.106027 & 0.008 \\
\hline idade & -0.03521 & 0.000 & -0.03511 & 0.000 & -0.03606 & 0.000 & -0.03584 & 0.000 \\
\hline idadeq & 0.000447 & 0.000 & 0.000445 & 0.000 & 0.000452 & 0.000 & 0.000449 & 0.000 \\
\hline casado & 0.11727 & 0.025 & 0.118083 & 0.025 & 0.107807 & 0.038 & 0.108358 & 0.039 \\
\hline divorciado & -0.26403 & 0.001 & -0.26797 & 0.002 & -0.27181 & 0.001 & -0.276 & 0.002 \\
\hline empregadopub & 0.184032 & 0.030 & 0.183929 & 0.042 & 0.172829 & 0.041 & 0.173248 & 0.055 \\
\hline empregadopriv & 0.21676 & 0.000 & 0.216988 & 0.000 & 0.191138 & 0.001 & 0.191144 & 0.000 \\
\hline desempregado & -0.54912 & 0.000 & -0.55032 & 0.000 & -0.54883 & 0.000 & -0.55009 & 0.000 \\
\hline aposentado & -0.02586 & 0.764 & -0.02448 & 0.757 & -0.03179 & 0.713 & -0.02989 & 0.709 \\
\hline donacasa & 0.005089 & 0.939 & 0.00292 & 0.961 & -0.00245 & 0.971 & -0.00459 & 0.939 \\
\hline estudante & 0.241342 & 0.032 & 0.241182 & 0.036 & 0.24779 & 0.026 & 0.247731 & 0.031 \\
\hline primario & 0.013972 & 0.813 & 0.012561 & 0.841 & -0.00961 & 0.870 & -0.0109 & 0.861 \\
\hline secundario & -0.09369 & 0.085 & -0.09344 & 0.115 & -0.11333 & 0.035 & -0.11345 & 0.060 \\
\hline superior & 0.255385 & 0.014 & 0.249257 & 0.016 & 0.228145 & 0.027 & 0.221078 & 0.030 \\
\hline c510 & -0.10494 & 0.400 & -0.10701 & 0.568 & -0.05074 & 0.683 & -0.05359 & 0.782 \\
\hline $\mathrm{c} 1020$ & -0.00783 & 0.944 & -0.01125 & 0.944 & 0.026523 & 0.809 & 0.022443 & 0.888 \\
\hline c2040 & -0.17955 & 0.100 & -0.1799 & 0.242 & -0.10628 & 0.321 & -0.10636 & 0.484 \\
\hline c4050 & -0.35736 & 0.002 & -0.35903 & 0.026 & -0.31064 & 0.007 & -0.3135 & 0.053 \\
\hline c50100 & -0.32261 & 0.002 & -0.32364 & 0.026 & -0.29903 & 0.003 & -0.29994 & 0.037 \\
\hline c100up & -0.52048 & 0.000 & -0.52185 & 0.000 & -0.45587 & 0.000 & -0.45728 & 0.002 \\
\hline capit & -0.24793 & 0.031 & -0.24858 & 0.085 & -0.2164 & 0.054 & -0.21702 & 0.128 \\
\hline tempo & -0.04241 & 0.005 & -0.04241 & 0.032 & 0.015651 & 0.080 & 0.015747 & 0.177 \\
\hline txinflap & -5.50319 & 0.005 & -5.69803 & 0.021 & $X$ & $\mathrm{X}$ & $\mathrm{X}$ & $\mathrm{X}$ \\
\hline txdesempp & -22.2911 & 0.000 & -22.5463 & 0.002 & $\mathrm{X}$ & $\mathrm{X}$ & $\mathrm{X}$ & $\mathrm{X}$ \\
\hline infregsul & 3.936674 & 0.285 & 4.220296 & 0.357 & $\mathrm{X}$ & $\mathrm{X}$ & $X$ & $X$ \\
\hline infregcoeste & 8.33712 & 0.098 & 8.815676 & 0.176 & $\mathrm{X}$ & $\mathrm{X}$ & $X$ & $\mathrm{X}$ \\
\hline infregnort & 0.848272 & 0.869 & 1.031028 & 0.854 & $\mathrm{X}$ & $\mathrm{X}$ & $\mathrm{X}$ & $\mathrm{X}$ \\
\hline
\end{tabular}

continua.. 
conclusão.

\begin{tabular}{lccccccccc}
\hline & \multicolumn{3}{c}{ Modelo I } & \multicolumn{5}{c}{ Modelo II } \\
\hline & Sem plano & \multicolumn{3}{c}{ Com plano } & & Sem plano & \multicolumn{3}{c}{ Com plano } \\
\hline infregnord & 5.780519 & 0.072 & 5.875901 & 0.126 & $X$ & $X$ & $X$ & $X$ \\
desregsul & -9.14775 & 0.142 & -8.84017 & 0.341 & $X$ & $X$ & $X$ & $X$ \\
desregcoeste & 1.866313 & 0.822 & 2.419775 & 0.856 & $X$ & $X$ & $X$ & $X$ \\
desregnort & 9.83371 & 0.255 & 10.05355 & 0.250 & $X$ & $X$ & $X$ & $X$ \\
desregnord & 9.59209 & 0.079 & 9.695544 & 0.176 & $X$ & $X$ & $X$ & $X$ \\
infreg & $X$ & $X$ & $X$ & $X$ & -0.01934 & 0.116 & -0.02008 & 0.217 \\
desreg & $X$ & $X$ & $X$ & $X$ & -0.00841 & 0.527 & -0.00936 & 0.634 \\
\hline
\end{tabular}

Fonte: Elaboração própria.

Recebido em: 19/03/2018.

Aceito em: 22/01/2020.

\section{(c) BY}


